\title{
Observing Vibrational Wavepackets during an Ultrafast Electron Transfer Reaction
}

Shahnawaz, Rafiq, Jacob C. Dean, Gregory D. Scholes*

Department of Chemistry, Princeton University, Princeton, New Jersey 08544 USA

Supporting information 
Figure S1. Narrowband transient absorption spectral data of oxazine 1 in chloronaphthalene $(\mathrm{Ox} 1 / \mathrm{CN})$ and dimethylaniline (Ox1/DMA) at various pump probe delay times shown on the right side of respective plots. The different spectral features are ground state bleach (GSB), excited state absorption (ESA) and photo-induced absorption (PIA) of oxazine 1 radical formed during electron transfer from DMA to Ox1. Arrow direction indicates the decay or growth of a particular signal.
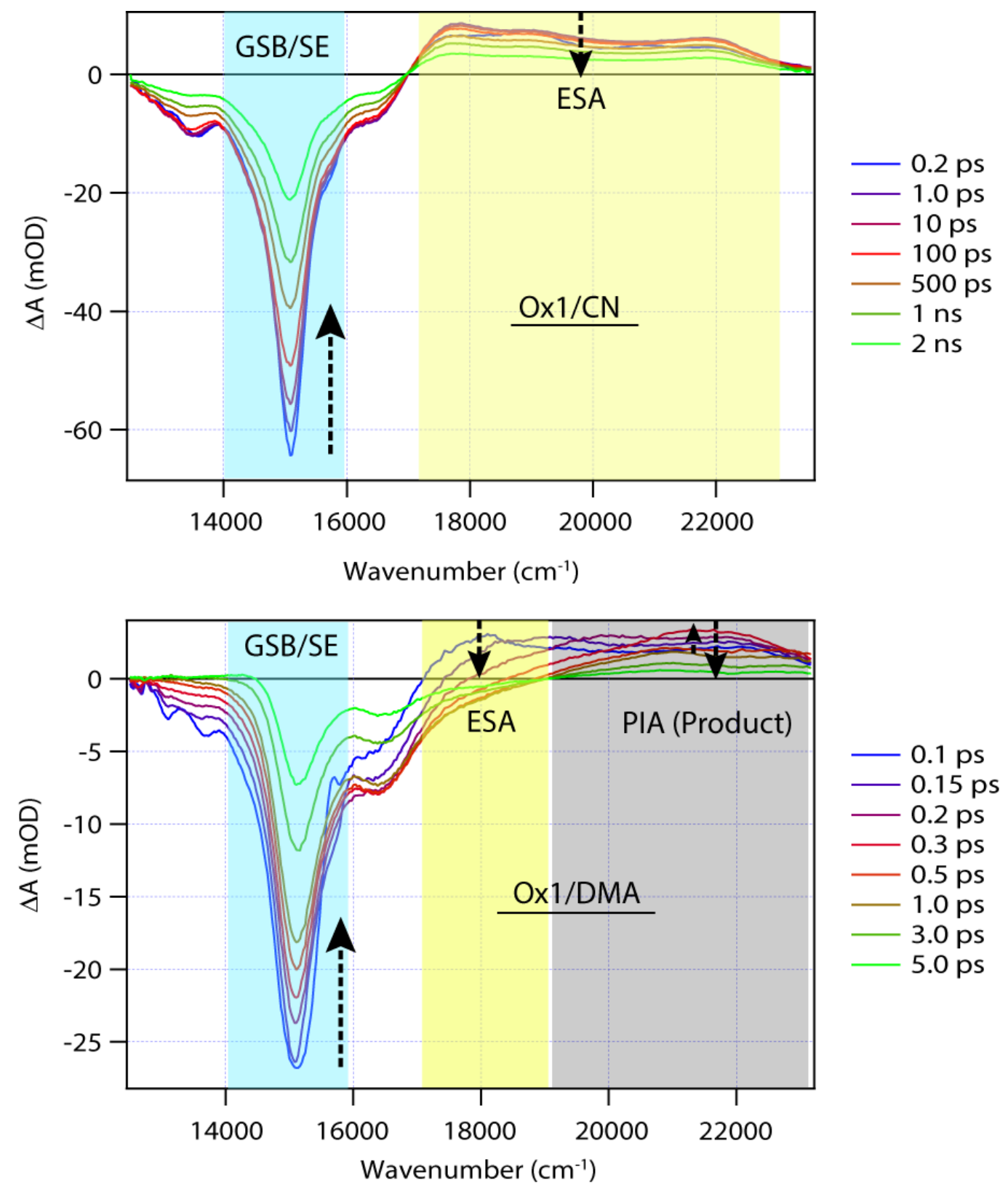
Figure S2. Single trace Fourier transform analysis of oxazine 1 in chloronaphthalene performed at different probe frequencies of the pump probe data. The left plot shows the oscillatory region from $450-700 \mathrm{~cm}^{-1}$ and right plot shows from $1000-1700 \mathrm{~cm}^{-1}$. The individual FT's are scaled to different values to see the oscillatory component properly.

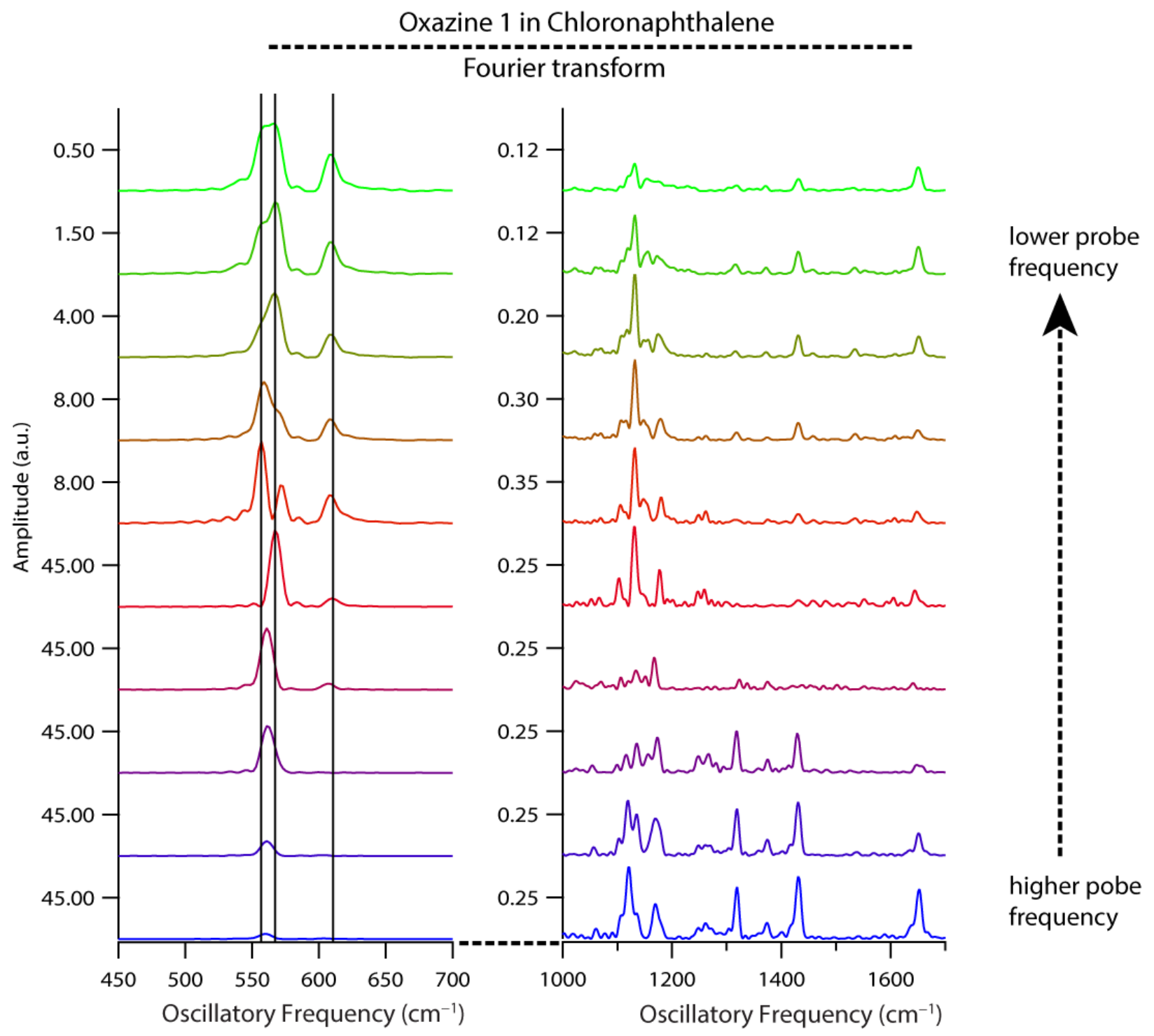


Figure S3. Pump probe spectra of Ox $1 / \mathrm{CN}$ measured with two laser spectra are shown here. The left panel shows bleach and stimulated emission around $15000 \mathrm{~cm}^{-1}$ and the right panel shows the excited state absorption around $17800 \mathrm{~cm}^{-1}$.

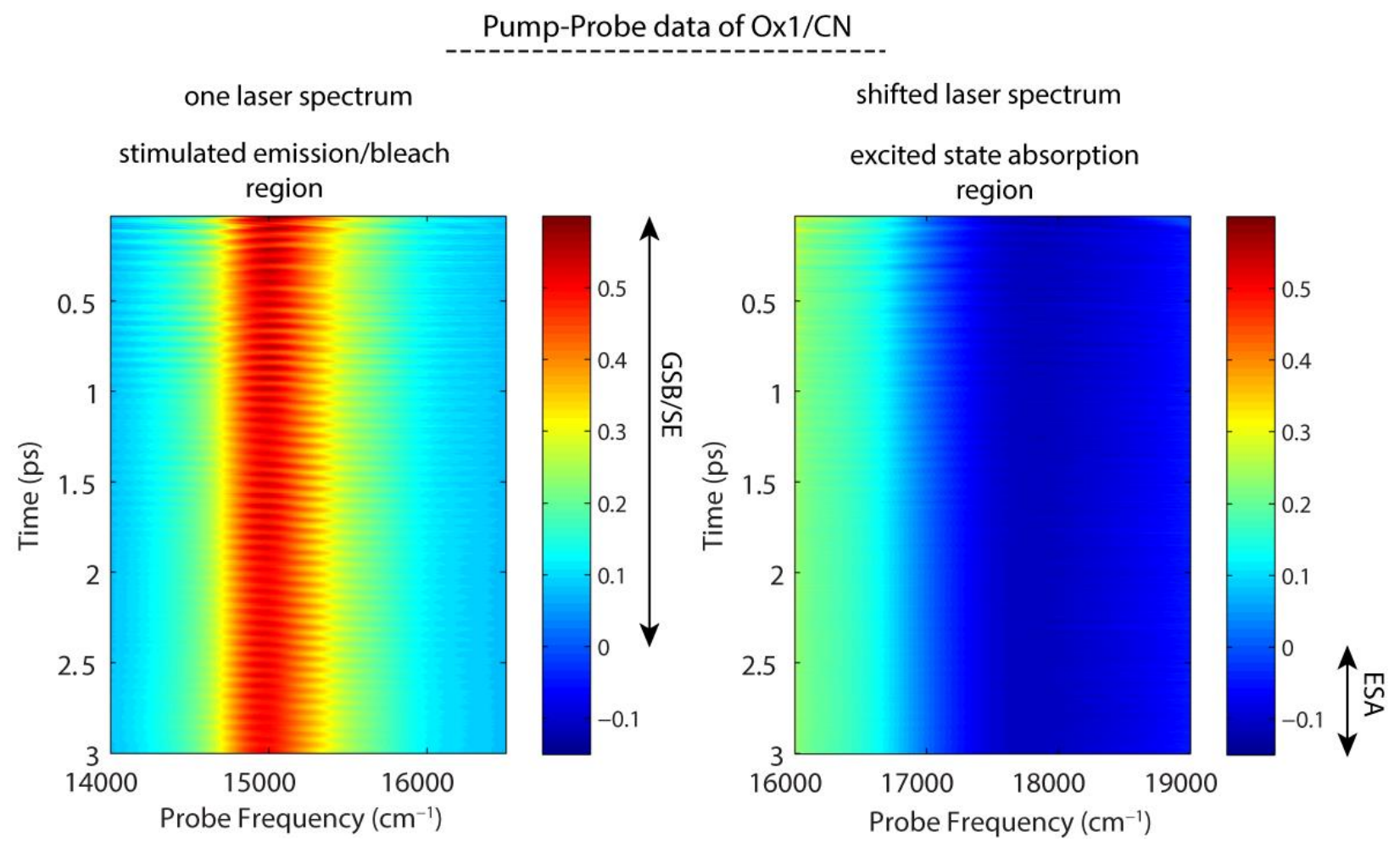


Figure S4. The time domain raw (top) and residual (middle) trace extracted at the excited state absorption maximum $\left(\sim 17850 \mathrm{~cm}^{-1}\right)$ in the pump probe data of oxazine 1 in chloronaphthalene. The bottom graph is the corresponding FT which show the oscillatory component a $561 \mathrm{~cm}^{-1}$. This dataset was obtained by using a shifted pulse spectrum.
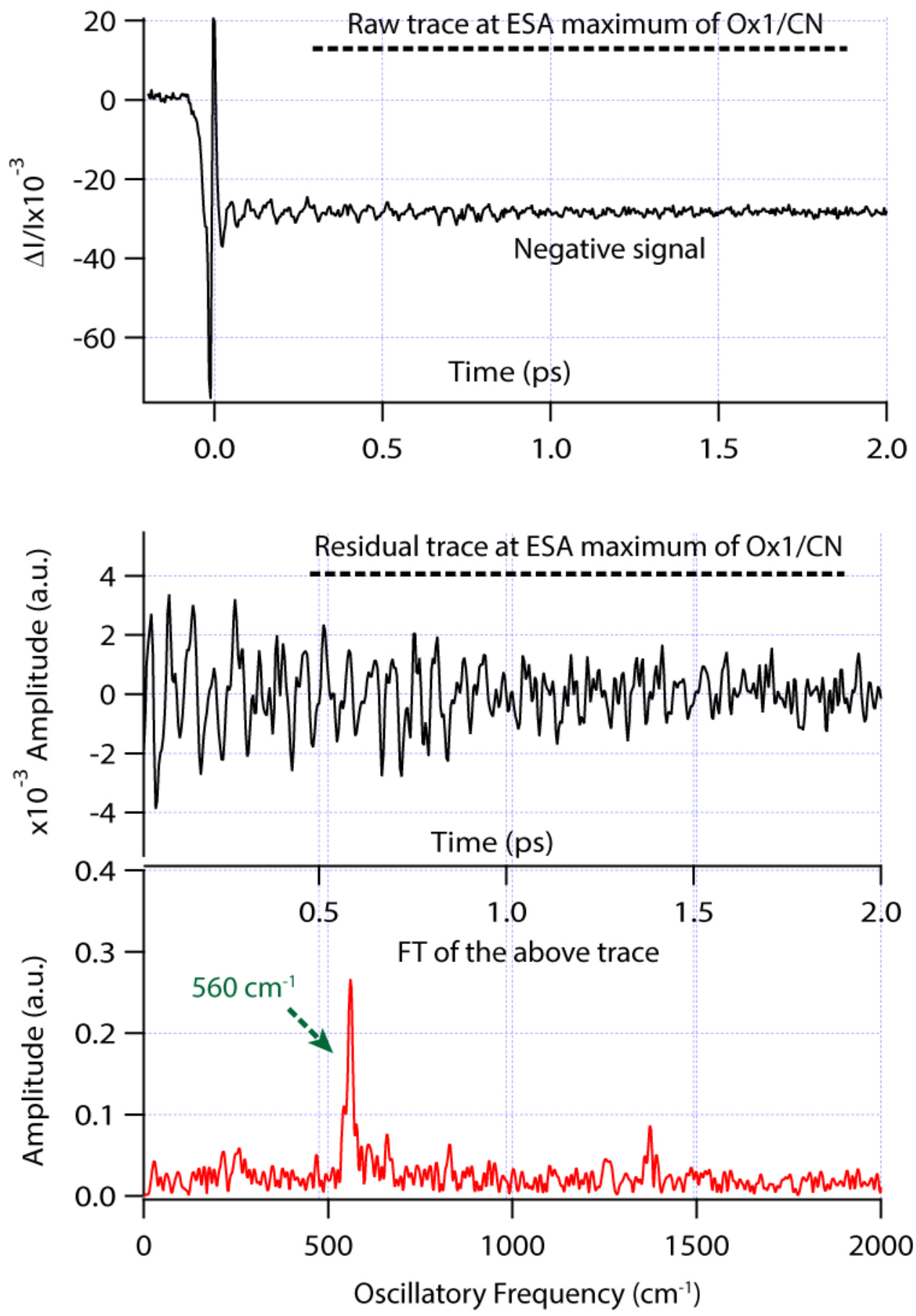
Figure S5. Pump probe spectrum of Ox1/DMA measured with two laser spectra are shown here. The left panel has mostly bleach signal around $15000 \mathrm{~cm}^{-1}$ and the right panel shows that the excited state absorption around $17800 \mathrm{~cm}^{-1}$ lasts for only about ca. $100 \mathrm{fs}$.

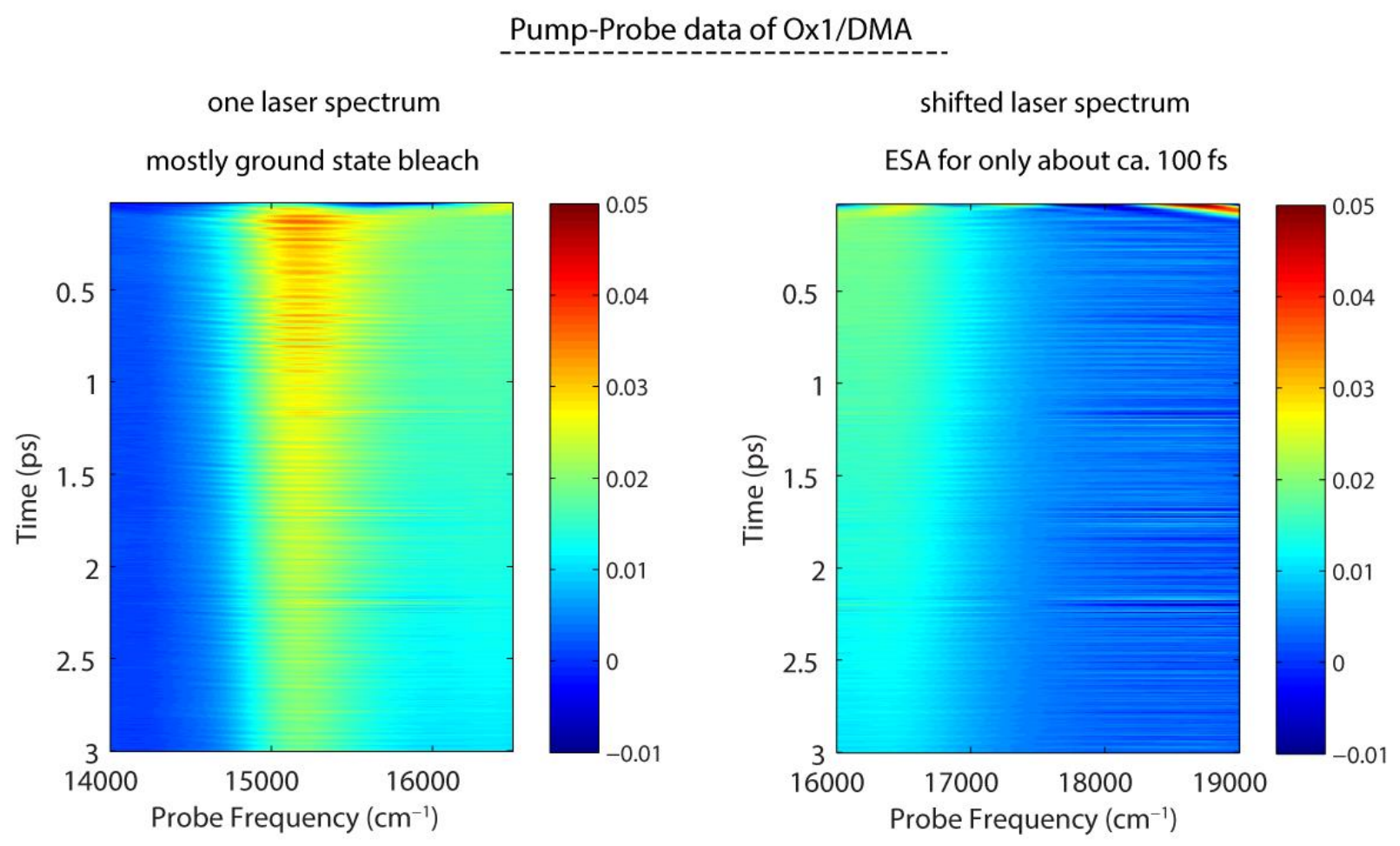


Figure S6. Single trace Fourier transform analysis of oxazine 1 in dimethylaniline performed at different probe frequencies of the pump probe data. The left plot shows the oscillatory region from $450-700 \mathrm{~cm}^{-1}$ and right plot shows from $1000-1700 \mathrm{~cm}^{-1}$. The individual FT's are scaled to different values to see the oscillatory component properly.

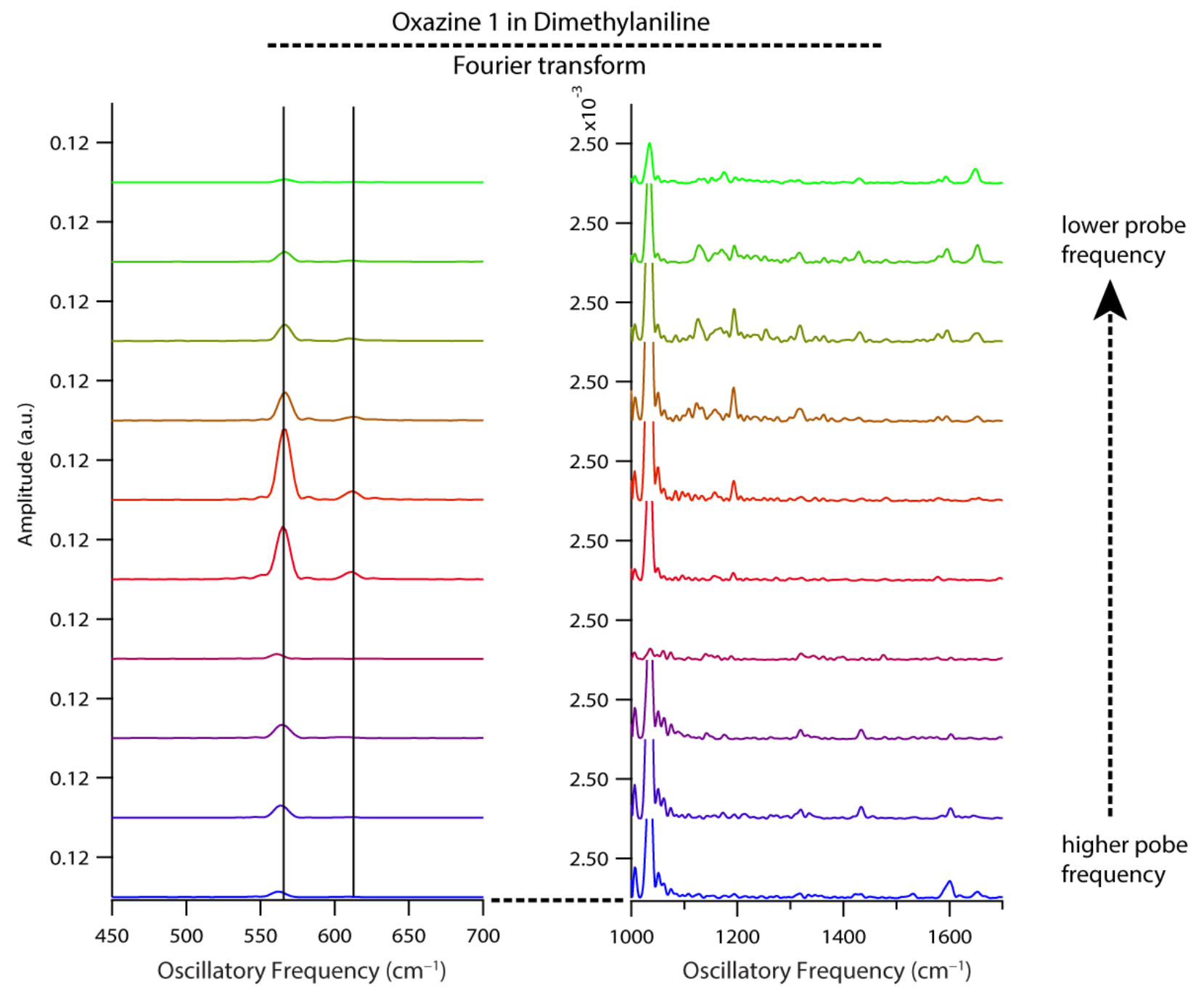


Figure S7. Area integrated kinetic traces extracted from 2D data of Ox1/DMA and neat DMA fitted with exponential function. Ox1/DMA was fitted with biexponential function giving two time constants; 5 fs and 100 fs. While as only one exponent was needed to fit the trace in DMA.
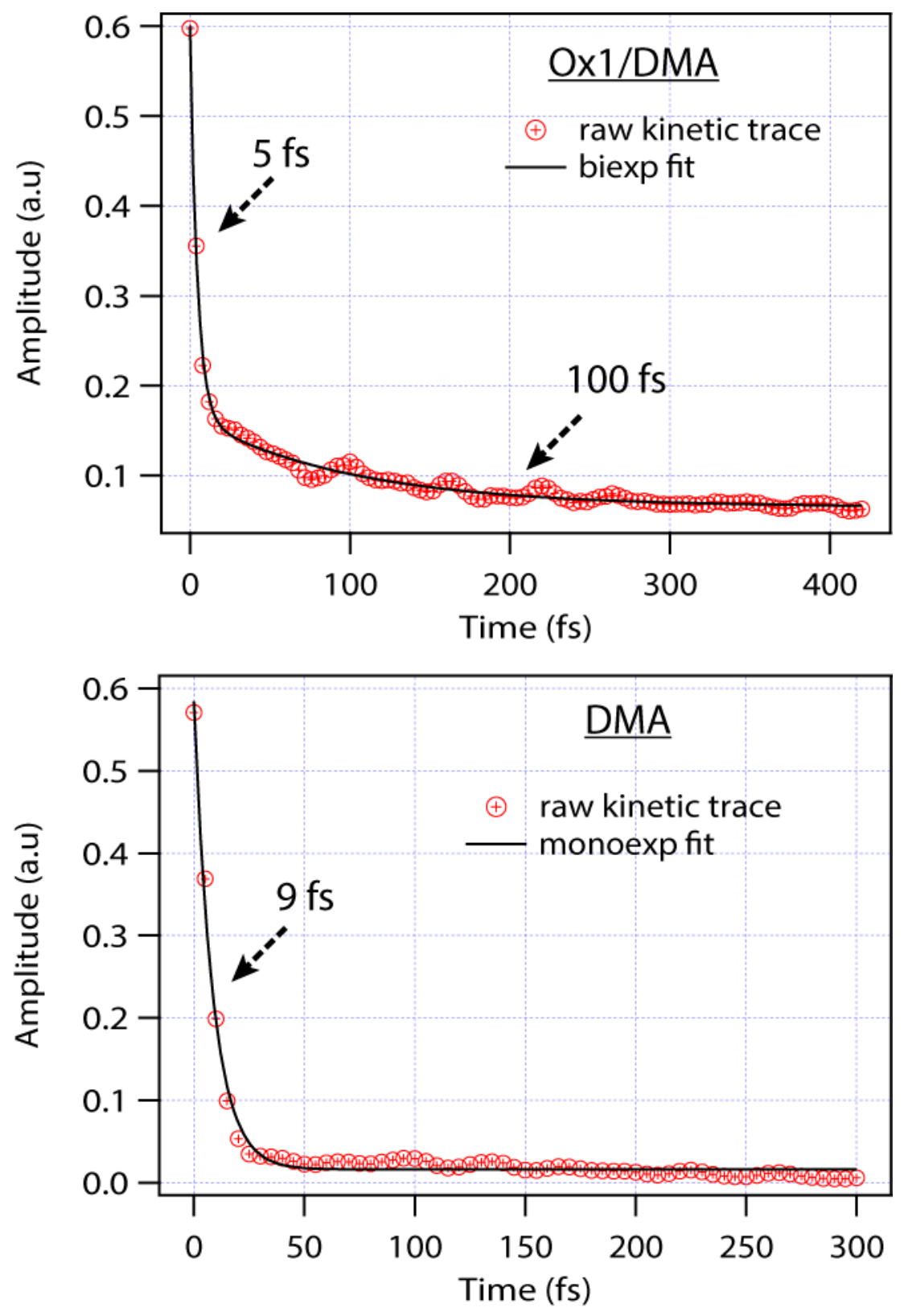
Figure S8. Fourier transform of 2D kinetic traces at the defined excitation - detection correlation frequencies shown by different colors on the 2D map in Ox1/CN and Ox1/DMA systems.

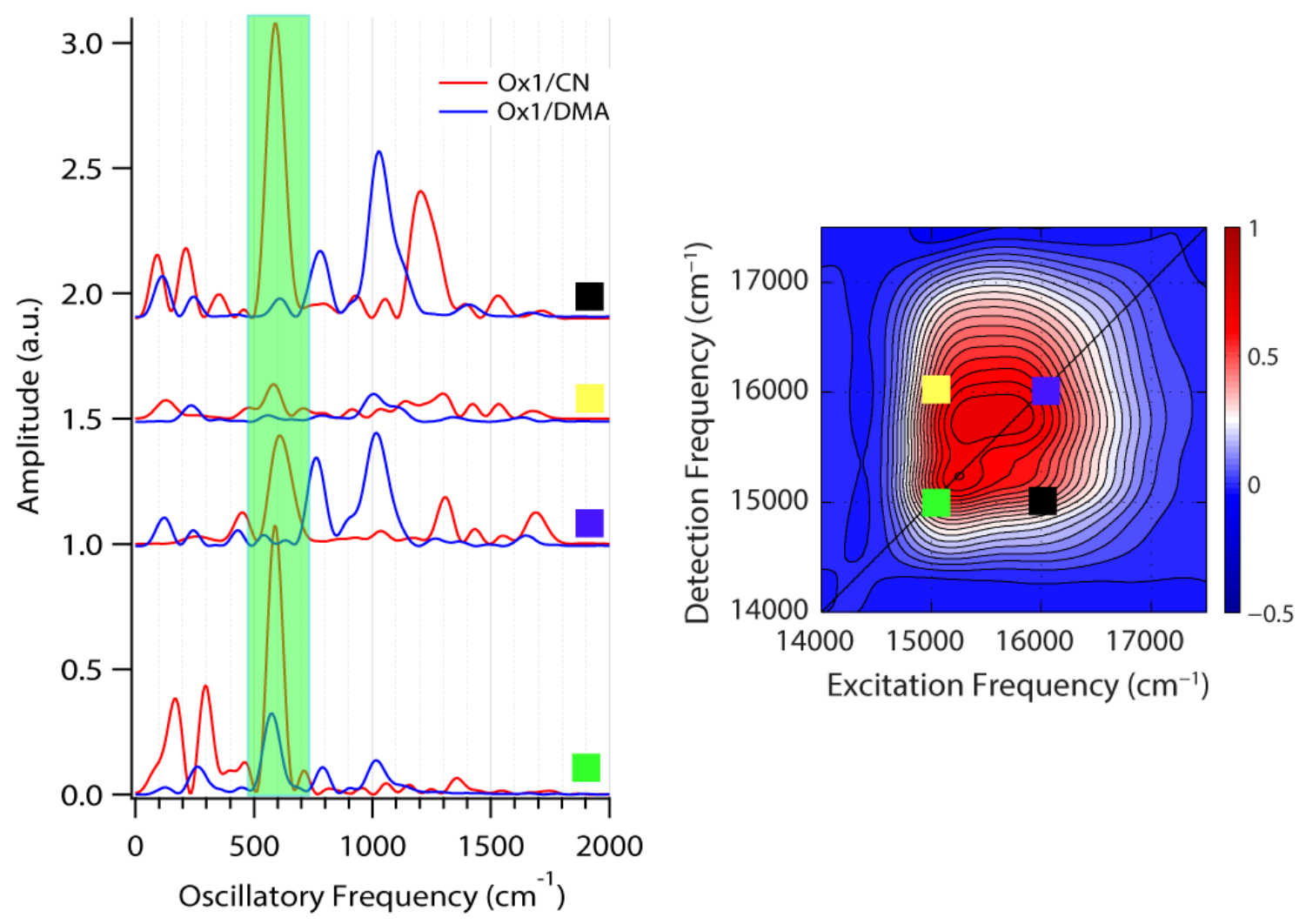


Figure S9. Fourier transform plot of the absorptive data set of oxazine 1 in chloronaphthalene (top graph) and dimethylaniline (bottom graph). These summary plots have been obtained by first performing FT at each point in the excitation - detection map and then summed along the excitation and detection axis. This gives a qualitative idea about all the oscillatory components in two dimensional data.

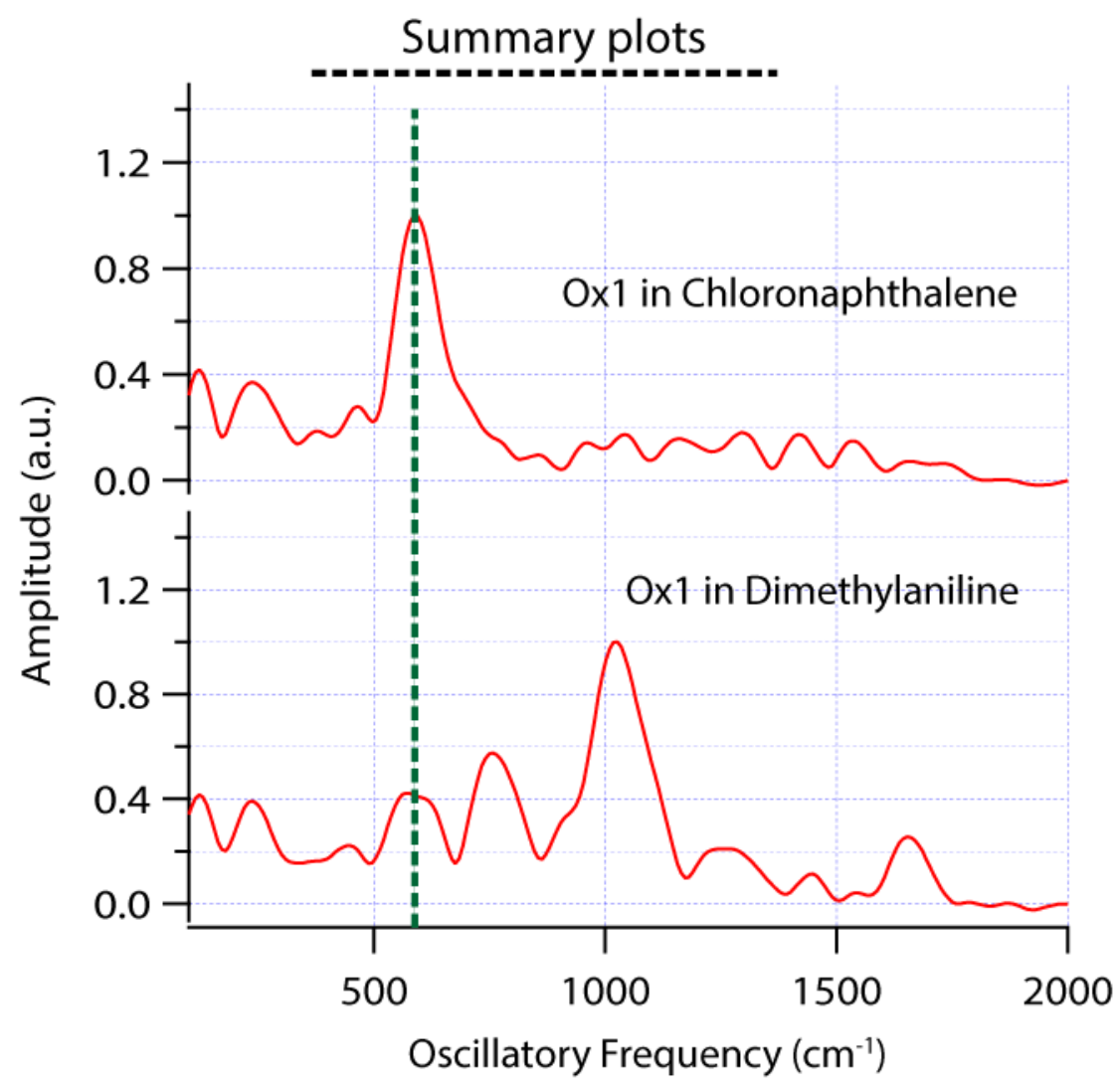

